\title{
Salidroside regulates imbalance of Th17/Treg and promotes ischemic tolerance by targeting STAT-3 in cerebral ischemia-reperfusion injury
}

Lele Yin ${ }^{1}$, Dongyun Ouyang ${ }^{2}$, Lihong Lin ${ }^{1}$, Xiufeng Xin ${ }^{3}$, Yuhua $\mathrm{Ji}^{2}$

\author{
${ }^{1}$ Department of Clinical laboratory, the First Affiliated Hospital of Jinan University, \\ Guangzhou, China \\ 2Department of Immunobiology, College of Life Science and Technology, \\ Jinan University, Guangzhou, China \\ ${ }^{3}$ Department of Neurology, the First Affiliated Hospital of Jinan University, Guangzhou, \\ China
}

Submitted: 23 January 2019; Accepted: 5 April 2019;

Online publication: 24 May 2019

Arch Med Sci 2021; 17 (2): 523-534

DOI: https://doi.org/10.5114/aoms.2019.85349

Copyright $\odot 2019$ Termedia \& Banach

\begin{abstract}
Introduction: The balance between Th17 and Treg cells controls the immune response and is an important regulator of helper T cells acting on autoimmune diseases. Focal cerebral ischemia-reperfusion injury can induce imbalance of Th17/Treg cells in the brain and the peripheral immune system of rats. The aim of this study was to investigate the effect of salidroside (Sal) on the ratio of Th17 and Treg cells in an adult rat model of middle cerebral artery occlusion (MCAO).

Material and methods: Forty rats were divided into 4 groups: normal group, sham group, surgery group, and Sal group. After treatment, the neurological deficits in rats were evaluated. Peripheral blood mononuclear cells were isolated and the count of Th17 and Treg cells was detected by flow cytometry. The infarct size and expression of ROR $\gamma \mathrm{t}$ and Foxp 3 were detected in rat brain tissue. Rat spleen cells were isolated, $C D 4^{+} \mathrm{T}$ cells were purified by immunomagnetic beads. Treg cells were induced by adding cytokine TGF- $\beta$. Th17 cells were induced by adding cytokine IL-6. The expression of STAT-3 was inhibited by SiRNA, and the effect of Sal on the differentiation of Th17/ Treg cells was analyzed. The expression levels of IL-6, TNF- $\alpha$, MCP-1, STAT-3 and NF- $\kappa-B 2$ proteins were examined.

Results: The results show that MCAO can induce an imbalance of Th17 and Treg cells in peripheral blood of rats. Sal treatment can significantly reduce the neurological deficit and infarct size of MCAO rats, reverse the oxidative stress of rat brain tissue, and inhibit the apoptosis of brain cells in MCAO rats. In the brain tissue of MCAO rats, Sal could significantly inhibit the expression of IL-6, TNF- $\alpha$, MCP-1, STAT-3 and NF- - -B2. Down-regulation of STAT-3 significantly reversed the therapeutic effects of Sal treatment.

Conclusions: Our results indicate that Sal can increase the tolerance of rat brain tissue to ischemia, inhibit cell apoptosis and reduce oxidative stress by targeting STAT-3.
\end{abstract}

Key words: salidroside, STAT-3, middle cerebral artery occlusion, Th17, Treg.

\author{
Corresponding authors: \\ Yuhua Ji \\ Department \\ of Immunobiology \\ College of Life Science \\ and Technology \\ Jinan University \\ Guangzhou 510632, China \\ Phone: $+86(20) 85227730$ \\ E-mail: yuhuaji138@163.com \\ Lele Yin \\ Department \\ of Clinical Laboratory \\ Center \\ the First Affiliated Hospital \\ of Jinan University \\ Guangzhou 510630, China \\ Phone: $+86(20) 38688400$ \\ E-mail: leleyin168@163.com.
}

\section{Introduction}

Ischemic cerebrovascular disease is a common clinical disease with high disability rate, high recurrence and slow recovery [1]. The mortality 
rate of ischemic cerebrovascular disease is second only to heart disease and is one of the major causes of death in humans [2, 3]. Focal cerebral ischemia-reperfusion injury is a complex pathological cascade. The factors affecting this pathological process are various, including the release of inflammatory mediators after ischemia, endothelial cell dysfunction, mitochondrial damage and oxygen failure $[4,5]$.

Th17 cells are $\mathrm{CD}^{+}{ }^{+} \mathrm{T}$ cells that secrete interleukin (IL) 17 specifically under the regulation of the nuclear transcription factor ROR- $\gamma \mathrm{t}$. IL-17 can promote the differentiation and maturation of neutrophils by inducing granulocyte colony-stimulating factor, stimulate the activation of monocytes and fibroblasts, and exert a potent pro-inflammatory effect $[6,7]$. Therefore, IL-17 is considered to be a key factor in inducing an inflammatory response and is involved in a variety of autoimmune diseases [8]. Th17 cells not only secrete IL-17, but also secrete cytokines such as IL-21 and IL-22, and participate in various autoimmune diseases. Treg cells are a subpopulation of $\mathrm{CD}^{+} \mathrm{T}$ cells with immunosuppressive activity [9]. Treg cells inhibit the production of inflammatory cytokines by releasing the cytokines IL-10 and TGF- $\beta$ to inhibit the function of $T$ cells and antigen presenting cells [10]. Foxp3 is an important transcription factor of Treg, and its sustained expression is a key factor in maintaining Treg inhibitory activity [11]. Treg exerts immunomodulatory effects by up-regulating the expression of inhibitory immune cell surface molecules and down-regulating the expression of genes involved in activated T cells [12]. Th17/Treg balance is important for focal cerebral ischemia/reperfusion injury. Once the Th17/Treg balance is destroyed, it will cause disease. Therefore, maintaining Th17/ Treg balance is important for the treatment of focal cerebral ischemia/reperfusion injury. Studies have shown that IL-17 can promote the secretion of IL-6, TNF- $\alpha$ and other pro-inflammatory factors, by antigen-presenting cells, and participate in and expand the host inflammatory response [13-15]. IL- 6 is a key cytokine that can determine the differentiation of naive T cells into Th17 or Treg cells. The process involved in the induction of Th17 cell differentiation may be closely related to the activation of the transcription factor STAT-3 [16].

Rhodiola is the dry rhizome of Rhodiola rosea $\mathrm{L}$., and salidroside (Sal) is the main active ingredient of Rhodiola [17]. In the rat model of focal cerebral ischemia/reperfusion injury, salidroside can reduce the infarct volume of the rat brain and reduce the neurological deficit score [18], but the exact mechanism is still unclear. The aim of this study was to investigate the effects of salidroside on the balance of Th17/Treg cells in rat brain tissue induced by focal cerebral ischemia-reper- fusion injury, as well as the expression levels of IL-6, TNF- $\alpha$, MCP-1, STAT-3 and NFK-B2 protein, and to further explore the mechanism of action of salidroside on neuroprotection in middle cerebral artery occlusion (MCAO) model rats.

\section{Material and methods}

\section{Experimental grouping and establishment of MCAO model rats}

The rats were purchased from the experimental animal centre of Southern Medical University. Forty rats were randomly divided into 4 groups - control group, sham group, MCAO group, Sal group with 10 rats in each group, and kept in cages. The establishment of MCAO model rats followed the following steps: rats were anesthetized by intraperitoneal injection of $10 \%$ chloral hydrate $(3 \mathrm{ml}$ kg body mass), methylpolysiloxane-coated nylon monofilaments were inserted from the left common carotid artery to the beginning of the middle cerebral artery. Two hours after embolization, the nylon monofilament was pulled out. The middle cerebral artery was not embolized in sham-operated animals. Before the operation, the rats were fed for 10 days to adapt to the environment, and the light was $12 \mathrm{~h}$ cycles during the day/night. At 3 days before the operation, the Sal group rats were given an intraperitoneal injection of Sal (Meilunbio, China) $12 \mathrm{mg} / \mathrm{kg}$ once a day, while the rest of the group was given an intraperitoneal injection of equal isotonic saline. During the operation, the temperature of the animal was maintained at $37^{\circ} \mathrm{C}$. On the second days after surgery, the peripheral blood mononuclear cells were isolated and the count of Th17 and Treg cells was detected by flow cytometry.

\section{Evaluation of neurologic deficit and detection of infarct size}

According to the Longa assessment [19], on the second day after surgery, the degree of neurologic deficit was evaluated in rats: grade 0 , no defect; grade 1, no extension of the lateral forelimb; grade 2, lateral forelimb flexion; grade 3 , mild reverse circle; grade 4, serious circle; grade 5 , contralateral paralysis. Rats were killed and brain tissues were collected. Some of them were frozen in liquid nitrogen and transferred to $-80^{\circ} \mathrm{C}$. The other part was cut into $3 \mathrm{~mm}$ by slicers and stained with $1 \%$ TTC for $30 \mathrm{~min}$. After staining, the film was scanned and photographed, and the infarct size was analyzed by ImageJ software.

\section{Apoptosis in rats' brain cells}

Rat brain tissue was cut into $4 \mathrm{~mm}$ slices and stained according to the TUNEL kit instructions 
provided by reagents (Beyotime, China). Five visual fields were randomly selected to count positive staining cells under a 100× microscope. The apoptosis was expressed by the ratio of TUNEL staining positive cells to the total number of cells in the field of view.

\section{Cell culture and siRNA interference}

Rat spleen cells of MCAO model rats were separated according to a method described in the literature [20]. The cytokine TGF- $\beta$ ( $2 \mathrm{ng} / \mathrm{ml})$ was added to induce Treg cells, and cytokines IL-6 $(20 \mathrm{ng} / \mathrm{ml})$ and TGF- $\beta(2 \mathrm{ng} / \mathrm{ml})$ were added to induce Th17 cells according to the reference materials [21]. The cells were divided into four groups: control group (control), the hypoxia group $(H)$, the hypoxia + Sal group (HS), the siRNA + hypox$\mathrm{ia}+\mathrm{Sal}$ group (SHS). The cells were cultured at $37^{\circ} \mathrm{C}, 5 \% \mathrm{CO}_{2}$ culture box. After the state became stable, the cells were used for gene silencing experimentation. Before receiving the drug treatment, the cells were treated with SATA-3 siRNA in accordance with the instructions provided by the manufacturer (Guangzhou Ribobio). The cells used in the hypoxia experiment were incubated with Sal $\left(10^{-4} \mathrm{~mol} / \mathrm{l}\right)$ for $24 \mathrm{~h}$ under the conditions of $1 \% \mathrm{O}_{2}, 5 \% \mathrm{CO}_{2}$, and $94 \% \mathrm{~N}_{2}$. The treated cells were divided into 3 parts: the first part was used to detect cell apoptosis, the second part of the cells was used to detect cell SOD and MDA, and the third part was used to detect the protein expression.

\section{MTT experiment}

The cell density was adjusted to $1 \times 10^{5} / \mathrm{ml}$ and inoculated with $100 \mu \mathrm{l}$ per hole in a 96-hole plate. After the cell growth reached $70 \%$ confluence, the cells were grouped by the above method. After the reoxygenation was finished, the cells were incubated with $20 \mu$ l of MTT (Beyotime, China) for $4 \mathrm{~h}$. After centrifugation the supernatant was discarded, then $150 \mu$ I DMSO was added to each hole and shaken for $10 \mathrm{~min}$. After the crystals were dissolved, the OD values of each group were detected at the wavelength $490 \mathrm{~nm}$.

\section{Flow cytometry}

For the expression of Th17 and Treg cells, peripheral blood mononuclear cells were isolated and the count of Th17 and Treg cells was detected by flow cytometry according to the reference [22]. For the apoptotic, after recovery, the cells were washed with PBS buffer 3 times. The cells were stained with FITC and PI for 10 min at room temperature. The apoptotic cells were detected by flow cytometry and the percentage of apoptotic cells was counted.

\section{Detection of cell SOD and MDA}

Changes of SOD activity and MDA content can sometimes occur due to acute stress and there is increased enzyme activity to eliminate free radicals that have arisen. After recovering the treated cells, the MDA in the medium was detected by the Lipid Peroxidation MDA Assay kit and SOD in the medium was detected with the Total Superoxide Dismutase Assay kit based on the instructions provided by the manufacturer. SOD activity and MDA content were detected with the method as reported previously [23]. All tests were repeated 3 times, and the average value of the test data was obtained.

\section{Western blot}

$50 \mathrm{mg}$ tissue samples or $10^{6}$ cells were taken from each sample. The total protein was extracted according to the instructions provided by reagents (Beyotime, China). A $60 \mu \mathrm{g}$ protein sample was taken for SDS-PAGE electrophoresis. The protein was transferred to the polyvinylidene fluoride (PVDF) membrane. The corresponding antibody was added to the TBST closed buffer to incubate overnight at $4^{\circ} \mathrm{C}$. The second antibody was added and incubated for $1 \mathrm{~h}$ at $37^{\circ} \mathrm{C}$. The results were observed after exposure to chemiluminescence. GAPDH was used as the internal reference, and Bio-image (Bio-Rad) analysis software was used to collect signals and grayscale scanning.

\section{Statistical analysis}

Statistical analysis was performed using SPSS 19.0 (IBM Corp., Armonk, NY, USA) and GraphPad PRISM version 5.00 (GraphPad Software, Inc., La Jolla, CA, USA) software. Data are presented as mean \pm standard deviation (SD), determined using single factor analysis of variance (ANOVA). Statistical significance was evaluated by one-way ANOVA followed by the least significant difference test or Dunnett's T3 post hoc analysis. $P<0.05$ was considered to indicate a statistically significant difference.

\section{Results}

\section{Sal can reduce neurological deficit and infarct size in MCAO rats}

As shown in Figure $1 \mathrm{~A}$, compared with the sham group, the MCAO group showed poor neurological function, while Sal could significantly reduce neurological deficit. The ischemic lesion area was not detected in the sham group, while in the MCAO group, the infarct size was significantly increased. However, after Sal pretreatment, infarct size decreased significantly (Figures $1 \mathrm{~B}, \mathrm{C}$ ). These results indicate that Sal can reduce infarct size induced by ischemia in rats. 

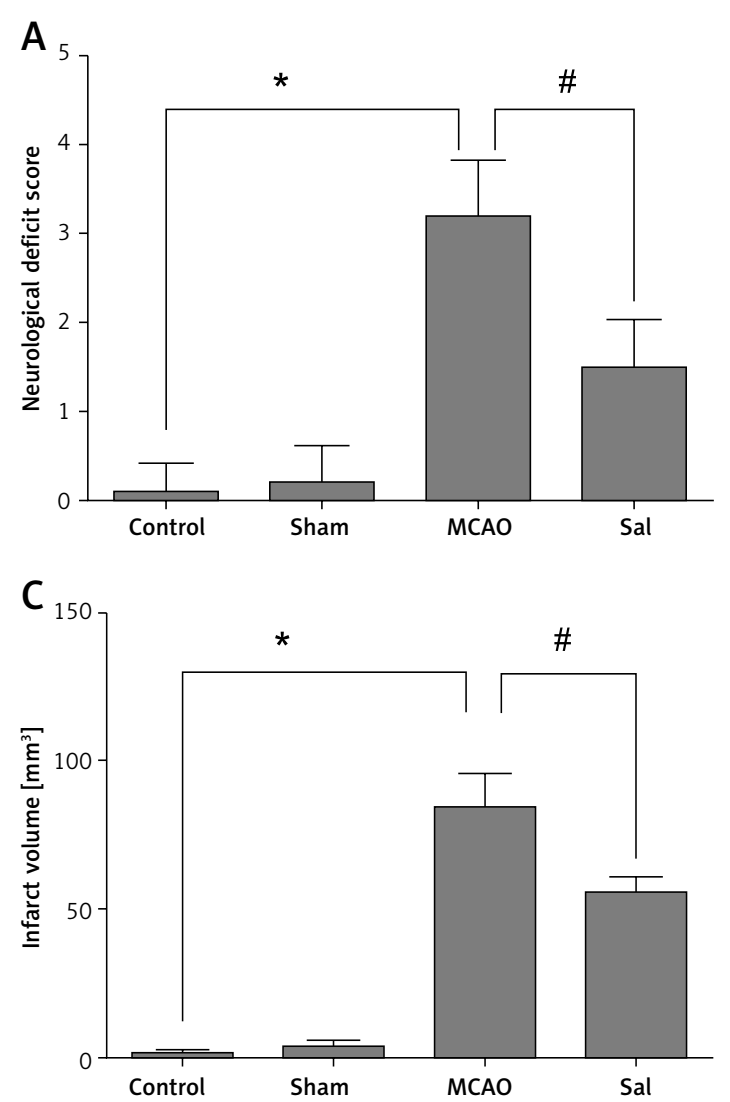

Sal can inhibit apoptosis of brain cells in MCAO rats

As shown in Figure 2, the apoptosis of brain cells in the MCAO group was significantly higher than that in the control group. However, the Sal treatment can significantly reduce the apoptosis of brain cells. These results indicate that Sal can inhibit the apoptosis of brain cells in MCAO rats.

\section{Sal can maintain the balance between Th17 and Treg cells}

Western blot results showed that the expression levels of ROR $\gamma$ t and Foxp3 protein in the brain tissue of the MCAO group were significantly higher compared with the control group (Figure 3), while the Sal group was opposite. These results suggest that MCAO operation can induce expression of the transcription factor ROR $\gamma$ t and Foxp3 protein in the rat neural system, and the changes in the expression of ROR $\gamma$ t and Foxp 3 protein directly lead to the change of the balance between Th17 and Treg cells. Also, Sal treatment can alleviate this trend.

As shown in Figure 4, the results of flow cytometry showed that MCAO could induce changes in the proportion of Th17 and Treg cells in the peripheral blood of rats, and Sal treatment can alleviate this trend.

\section{B}

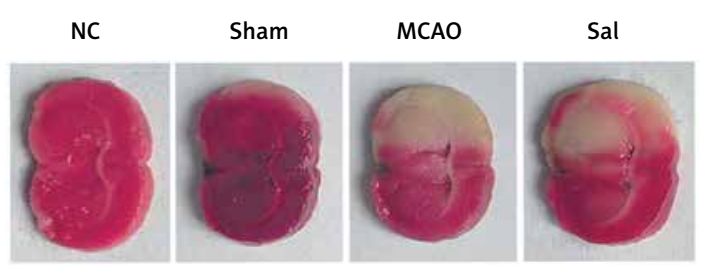

Figure 1. Sal can reduce the neuronal damage after MCAO. A - Neurological deficit score in rats after MCAO. B - Infarct volume was detected from ischemic rat brains. The white indicates infarction

Compared with the control group. ${ }^{*} p<0.05$; compared with MCAO group, ${ }^{*} p<0.05$.

\section{Successful construction of STAT-3 low expression cell line}

The initial T cells were induced by IL-6 and TGF- $\beta$, and then Thl7/ Treg cells were obtained. The expression of transcription factor STAT-3 was downregulated by siRNA. The results showed that the expression of STAT-3 gene in T cells was inhibited successfully (Figure 5).

\section{Sal can reduce the oxidative stress of $\mathrm{T}$ cells mediated by STAT-3}

As shown in Figure 6, the results showed that Sal treatment could significantly increase the activity of SOD (Figure $6 \mathrm{~A}$ ) and reduce the content of MDA (Figure 6 B). While the expression of STAT3 was inhibited, the effect of Sal was inhibited as well. These results showed that Sal probably can reduce the oxidative stress of $T$ cells, which may be mediated by STAT-3.

Sal can reduce the apoptosis of T cells and induce proliferation mediated by STAT-3

As shown in Figures $7 \mathrm{~A}$ and $\mathrm{B}$, hypoxia treatment could significantly increase the apoptosis of $T$ cells, and Sal treatment could significantly reduce the apoptosis of $\mathrm{T}$ cells after hypoxia treatment, while expression of the STAT-3 gene in T cells was inhibited, and Sal treatment lost its effect. 
Control

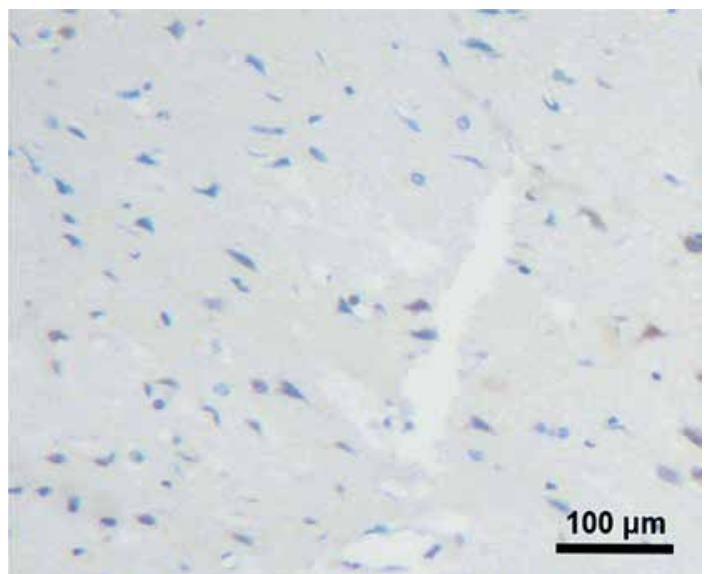

MCAO
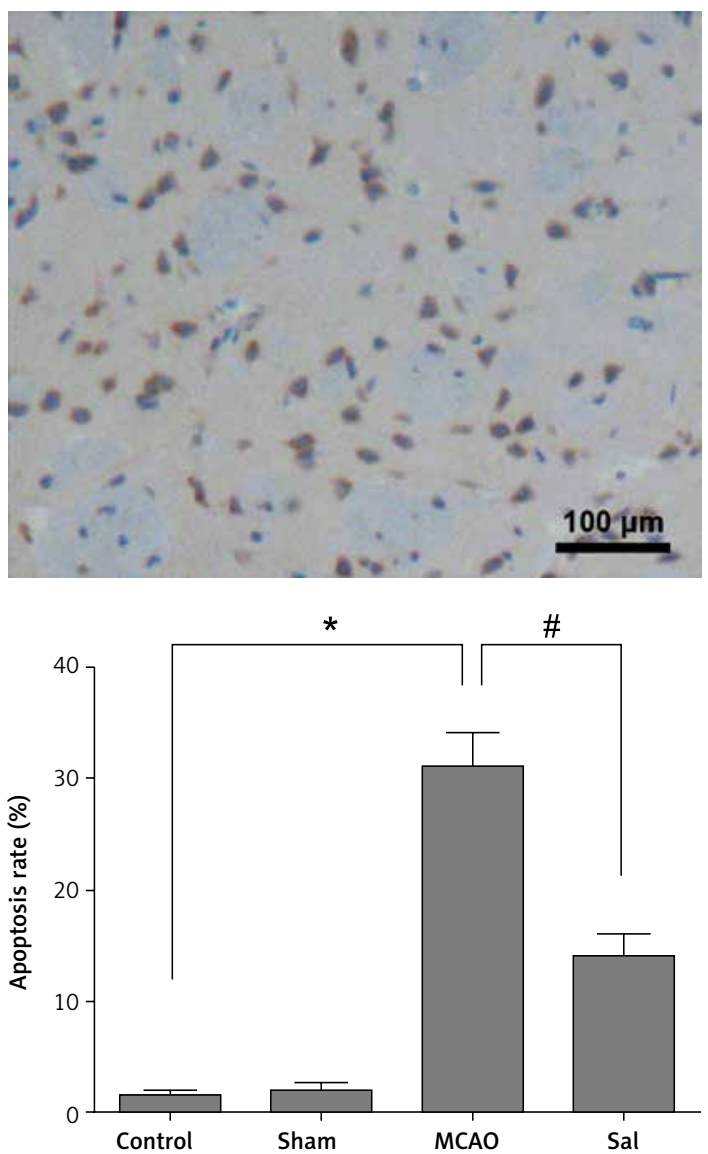

As shown in Figure $7 \mathrm{C}$, hypoxic treatment can significantly inhibit the activity of T cells, but after Sal treatment, cell viability increased significantly. However, it is interesting to note that after STAT-3 gene expression was inhibited, the role of Sal was also inhibited. These data indicate that Sal can promote the growth of T cells after anoxia treatment, which may be mediated by STAT-3.

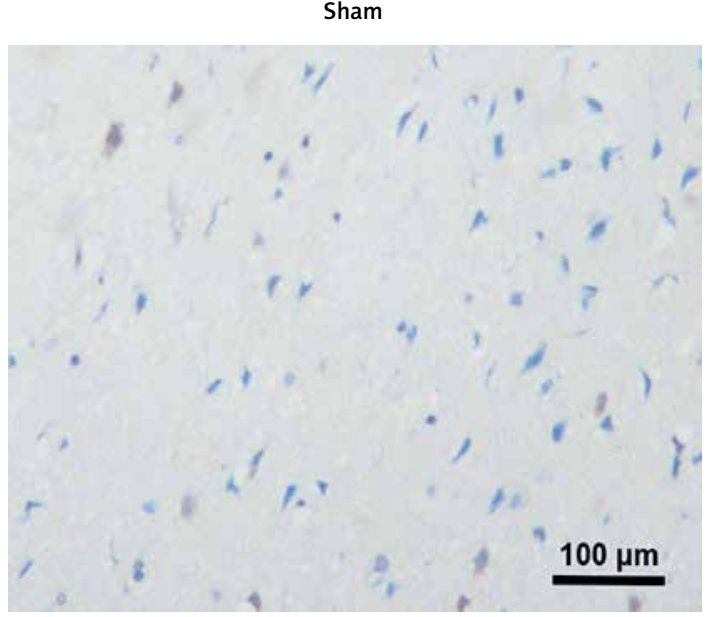

Sal

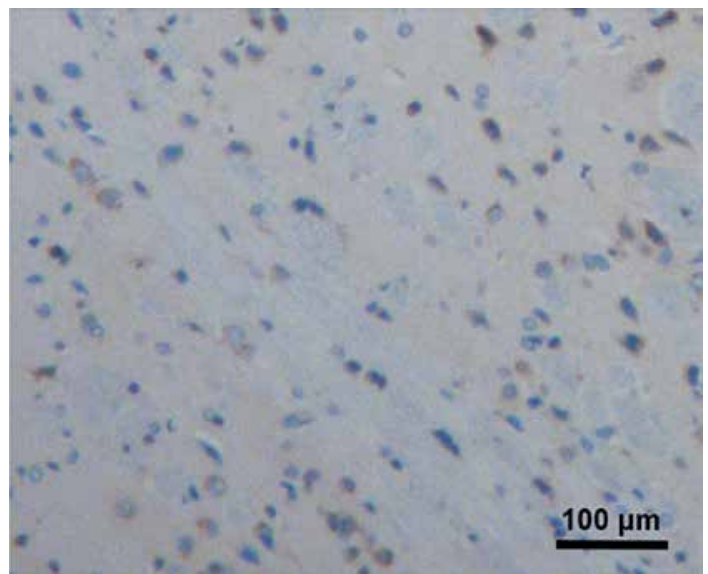

Figure 2. Apoptosis was detected with the TUNEL assay in rats' brain cells. Compared with control groups, MCAO group rats showed a significant increase in apoptosis, but the opposite results appeared in Sal groups. Dark brown represents apoptotic cells and the blue denotes nuclei

Compared with the control group, ${ }^{*} p<0.05$; compared with MCAO group, ${ }^{*} p<0.05$.

\section{Sal can inhibit the expression of IL-6, TNF- $\alpha$, MCP-1 and NF-к-B2 protein by targeting STAT-3}

Western blot was used to detect the expression of IL-6, TNF- $\alpha$, MCP-1, STAT-3 and NF- - -B2. The results showed that (Figure 8) Sal could significantly inhibit the expression of IL-6, TNF- $\alpha$, MCP-1, STAT-3 and NF- $\kappa-B 2$ protein in T cells after hypoxia treatment. However, this trend was offset by the knockdown of the STAT-3 gene. These data further support our hypothesis that Sal promotes 

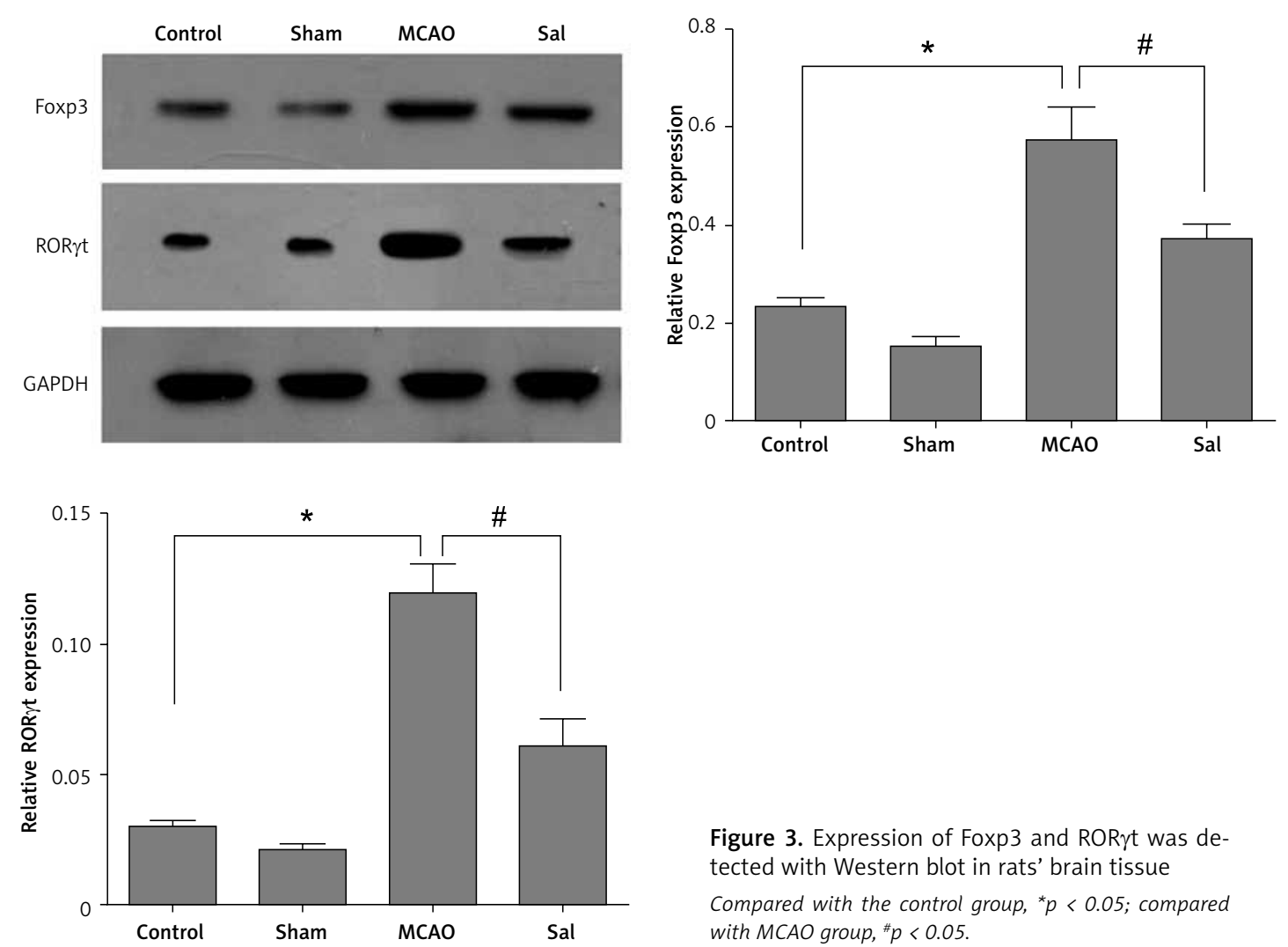

Figure 3. Expression of Foxp3 and RORyt was detected with Western blot in rats' brain tissue Compared with the control group, ${ }^{*} p<0.05$; compared with MCAO group, ${ }^{*} p<0.05$.
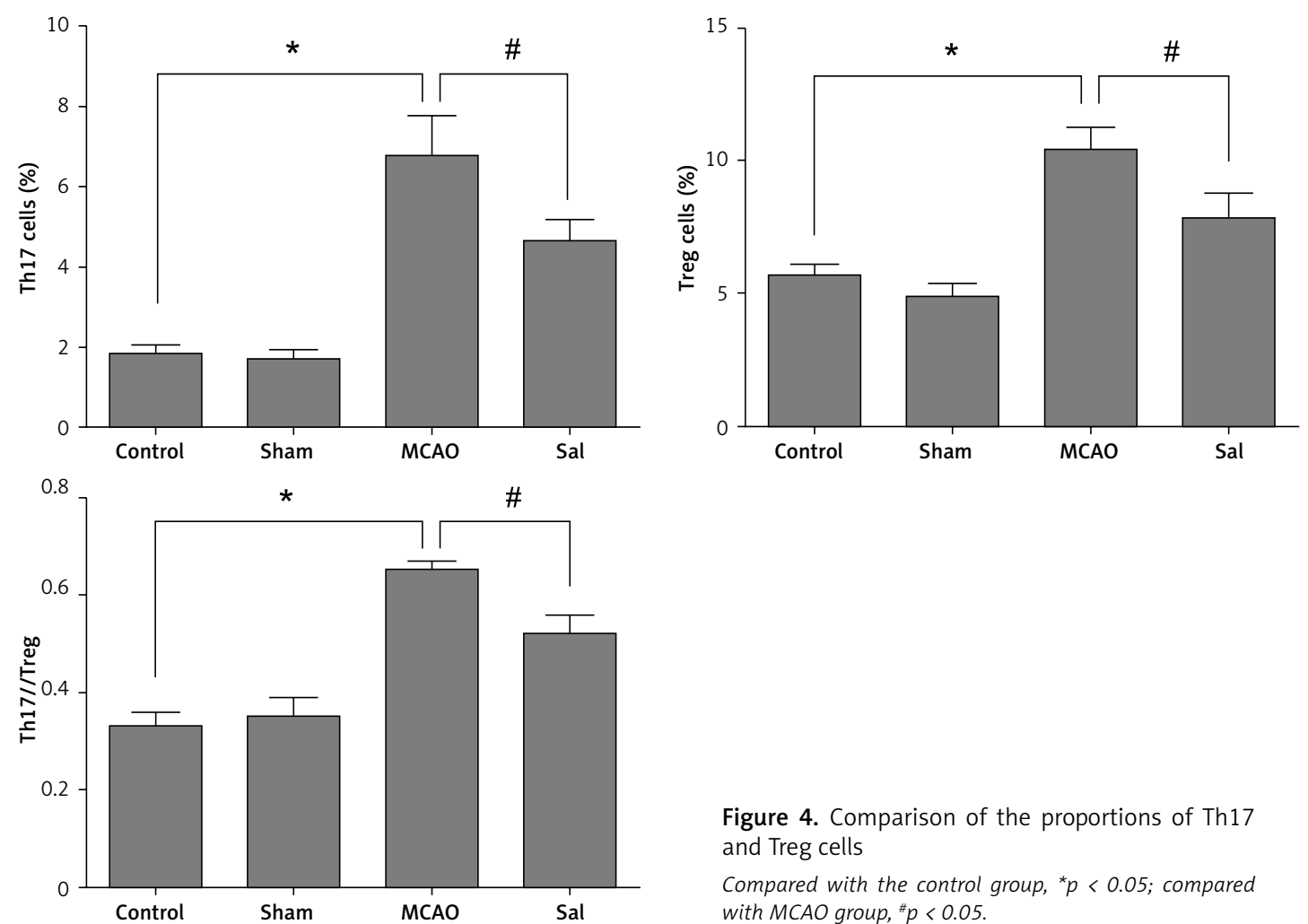

Figure 4. Comparison of the proportions of Th17 and Treg cells

Compared with the control group, ${ }^{*} p<0.05$; compared with MCAO group, ${ }^{*} p<0.05$. 


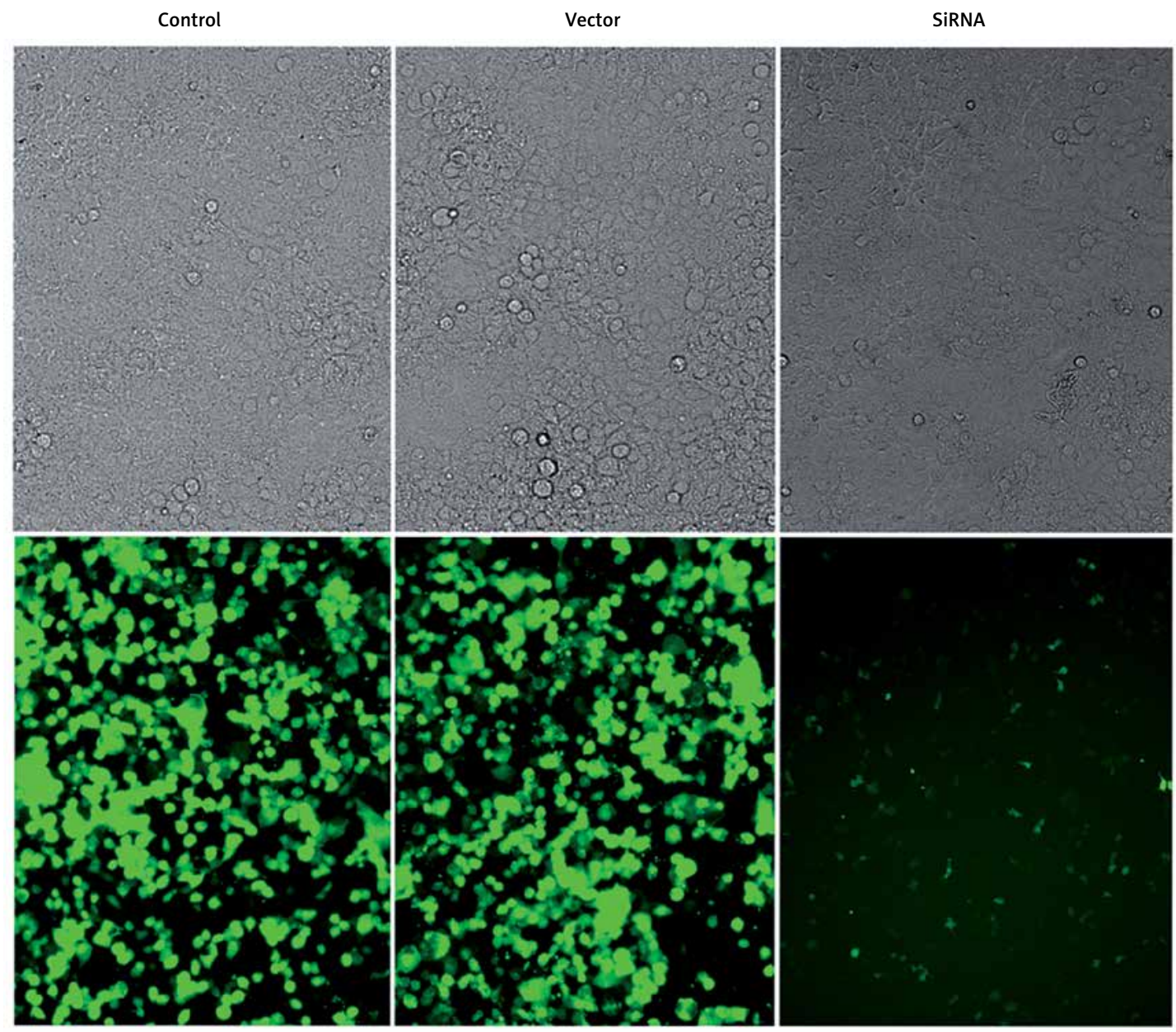

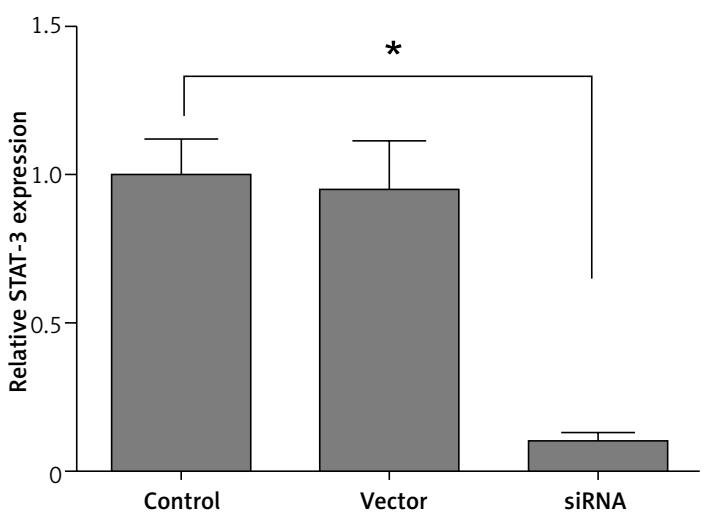

ischemic tolerance, and inhibits apoptosis and oxidative stress by targeting STAT-3.

\section{Discussion}

Salidroside has the effects of anti-hypoxia, anti-inflammatory, anti-apoptosis, anti-cancer and so on $[24,25]$. In this study, we detected the effects of salidroside on oxidative stress in the brain tissue of rats. The results showed that Sal could reduce the neurologic deficit in MCAO rats and reduce the infarct area caused by local ischemia in rats. Cerebral ischemia can significantly induce
Figure 5. The T cells with STAT-3 gene silencing was constructed. The expression of STAT-3 was evaluated using fluorescence microscopy in T cells after STAT-3 gene silencing

Compared with the control group, ${ }^{*} p<0.05$.

apoptosis of brain cells. This conclusion has been further confirmed in our experiment. But in this study, we also found that the Sal treatment can alleviate this trend. MDA level and SOD activity indirectly reflected oxidative stress. In vitro data showed that Sal could reduce MDA content and enhance SOD activity in T cells. In addition, our results indicate that Sal treatment can significantly inhibit the apoptosis of ischemic brain cells. These results indicate that Sal has significant protective effects of brain injury induced by ischemia in rats. But the mechanism is not yet clear. 

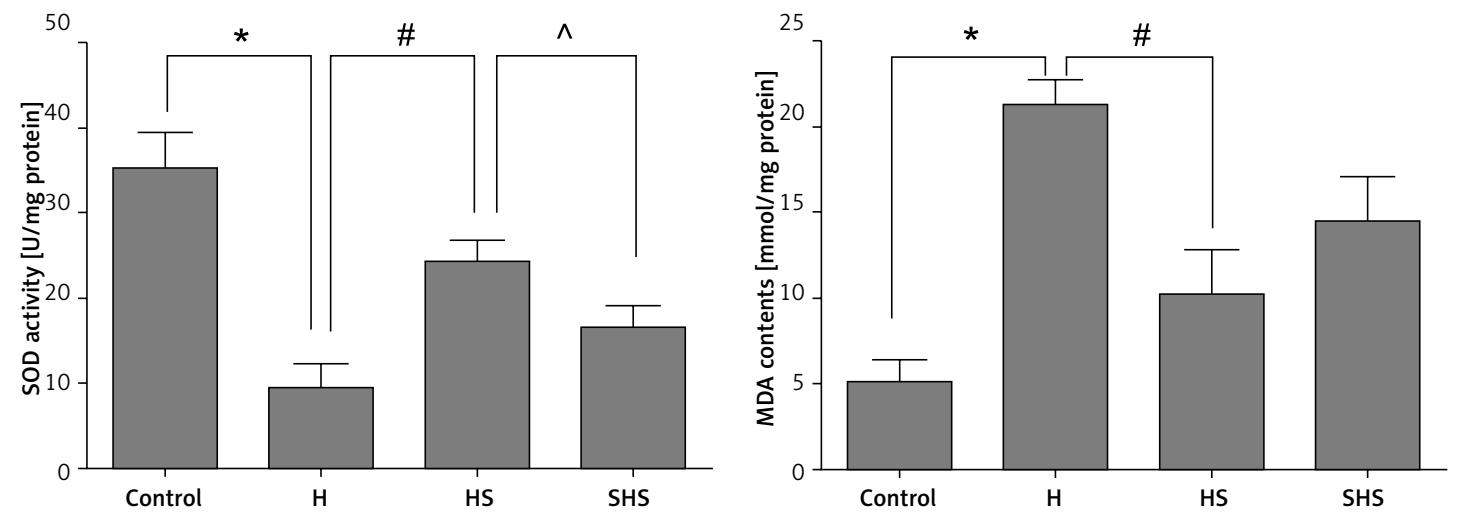

Figure 6. Assessment of oxidative states using assays of SOD and MDA in T cells after STAT-3 gene silencing $H$ - hypoxic condition, HS - hypoxic condition plus Sal, SHS - hypoxic condition plus Sal after STAT-3 gene silencing. Compared with the control group, ${ }^{*} p<0.05$; compared with H group, ${ }^{*} p<0.05$; compared with HS group, ${ }^{\wedge} p<0.05$.

The Th17 cell is a CD4+ cell that can secrete IL-17 specifically under the regulation of nuclear factor ROR- $\gamma$ t. IL-17 can promote the secretion of IL-6, TNF- $\alpha$ and many other proinflammatory cytokines in the antigen presenting cells, and raise the concentration of granulocytes to the inflammatory sites, and participate in and expand the host inflammatory response [26]. Treg cells are also known as regulatory T cells. Cytokines TGF- $\beta$ and IL-2 play a key role in the differentiation of Treg cells. TGF- $\beta$ is the first discovered cytokine that can induce peripheral differentiation of Treg cells [27, 28]. As an important T cell growth factor, IL-2 plays a central role in thymic differentiation of Treg cells. IL-2 can activate the JAK-STAT5 pathway [29]. STAT5 can directly bind to the Foxp3 gene to promote Foxp3 gene transcription, thereby promoting Foxp3 expression and inducing Treg cell differentiation [30]. The differentiation and regulation of Thl7 and Treg cells are completed under their respective inducible factors and cell pathways, and the differentiation process shows a complex and fine network relationship [31]. The subgroups are resistant and balanced to each other through their respective transcription factors and cytokines. It can be seen that there is a balance between Thl7 and Treg cell subsets in the normal state of the body. Once the balance is destroyed, it will lead to the occurrence of immune diseases [32]. Maintaining Thl7/Treg cell balance is of great importance to the immune homeostasis and the treatment of diseases. The balance of ROR- $\gamma$ t and Foxp3 is an important way to maintain the balance of Thl7/Treg cells. Therefore, in this study, we detected the expression level of ROR- $\gamma$ t and Foxp3 protein in the brain tissue of MCAO rats by Western blot. The numbers of Th17 and Treg cells in peripheral blood of each group were detected by flow cytometry. Our experimental data showed that the expression of ROR- $\gamma$ t and Foxp3 protein can be significantly induced by cerebral ischemia in rats, and the Sal treatment can reverse this result. The number of Th17 and Treg cells in the peripheral blood of rats was detected by flow cytometry. The results showed that the MCAO operation could induce a change of Th17 and Treg cells in the peripheral blood of rats, which resulted in the imbalance of Th17 and Treg cells, but Sal could relieve the ratio of Th17 and Treg cells in the peripheral blood of the rats. In conclusion, the protective effect of Sal on brain injury induced by ischemia in rats may be achieved by regulating the proportion of Th17 and Treg cells. It has been reported that the dynamic balance of transcription factor STAT3/STAT5 can represent the balance between Thl7/Treg cells [33]. However, the relationship between STAT3/STAT5 and ROR- $\gamma$ t/Foxp3 is not clear. In this study, STAT3 was used as a further research object, and specific siRNA was used to inhibit the expression of STAT3 in T cells. The results showed that Sal could significantly reduce the apoptosis of $T$ cells after hypoxia treatment, and the downregulation of STAT3 expression could inhibit the effect of Sal. This result suggests that the protective effect of Sal to hypoxia may be mediated by STAT3. In addition, we detected the proliferation characteristics of $T$ cells. The results showed that Sal could promote the proliferation of hypoxic cells, while the knockdown of STAT3 could counteract the effect of Sal. Sal can significantly inhibit the expression of T cells IL-6, TNF- $\alpha$, MCP-1, STAT-3 and NF- $\kappa$-B2 protein after hypoxia treatment. However, this state can be reversed by the knockdown of the STAT-3 gene. Studies have shown that STAT-5 can reduce the expression of ROR $\gamma$ t and antagonize the differentiation and survival of Thl7 cells by competitive inhibition of STAT-3 [34]. However, in this study, we did not knock down the expression of the STAT-5 gene in T cells, and thus failed to verify the relationship between STAT-3/STAT-5 in the process of brain damage induced by Sal reduction by hypoxia. These studies will need further exploration in the future. 
A

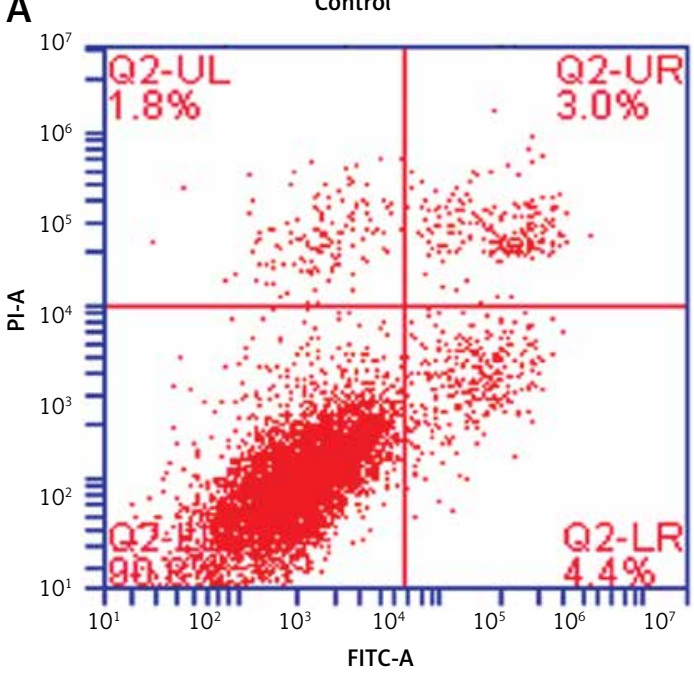

HS

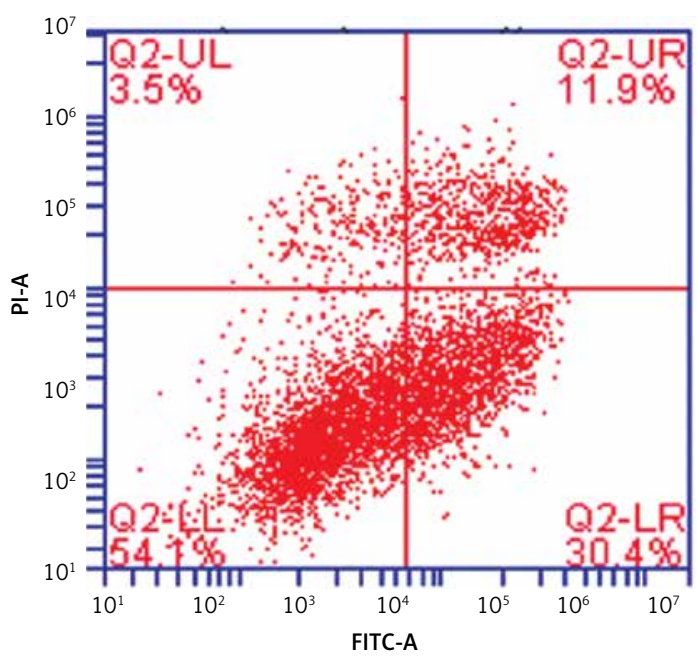

B

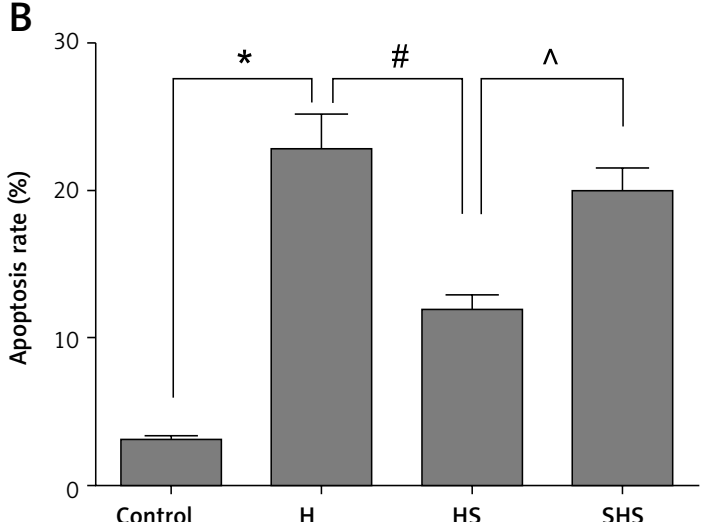

$\mathrm{H}$

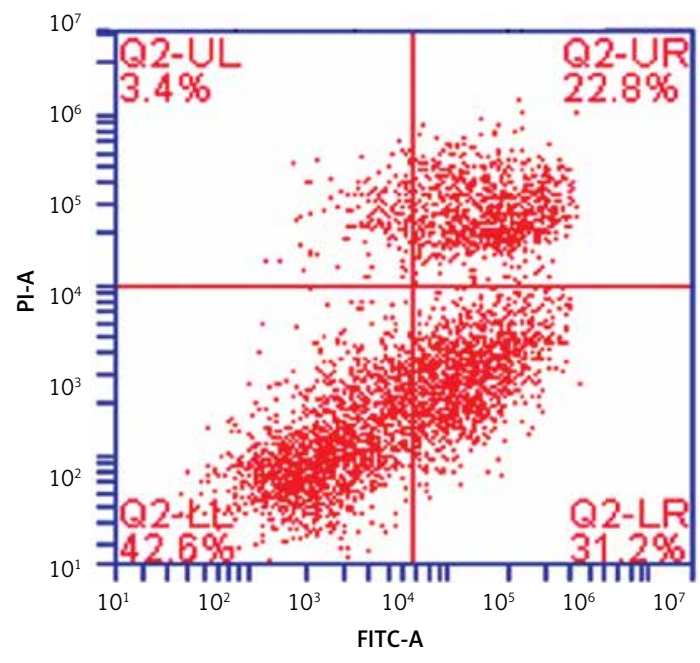

SHS

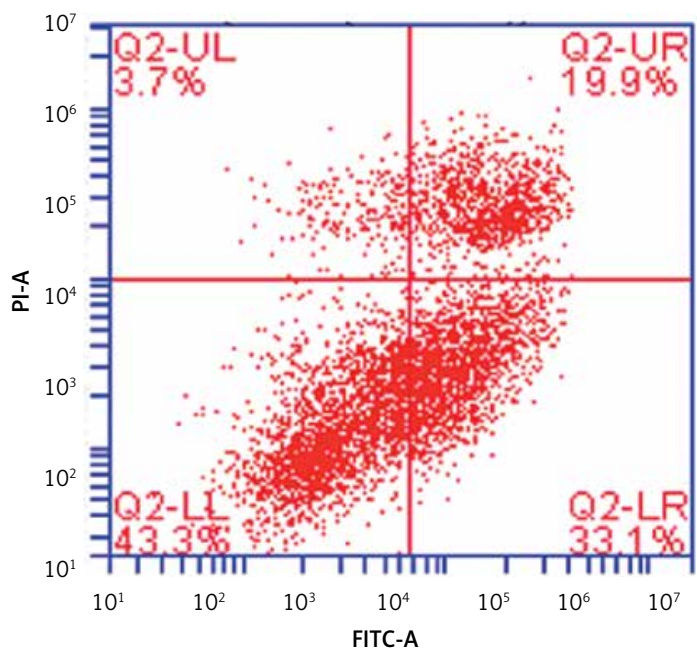

C

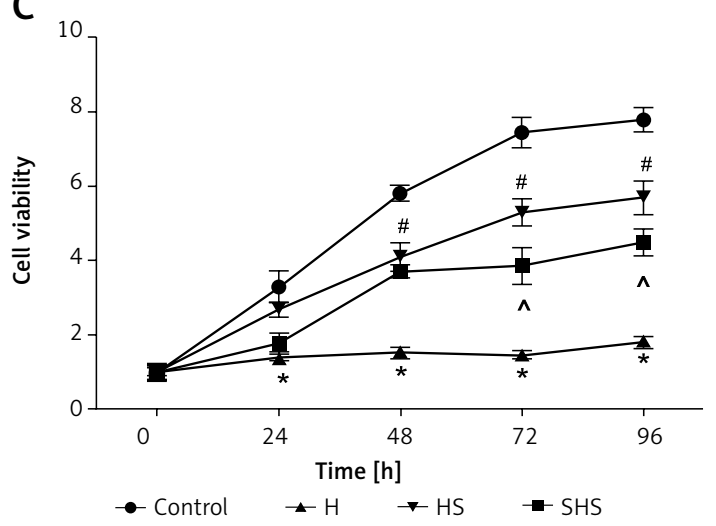

Figure 7. Apoptosis and proliferation of T cells after STAT-3 gene silencing. A, B - Apoptosis results of T cells treated under hypoxic conditions, Sal and STAT-3 gene silencing. C - MTT assays were performed to determine the proliferation in T cells

H - hypoxic condition, HS - hypoxic condition plus Sal, SHS - hypoxic condition plus Sal after STAT-3 gene silencing. Compared with the control group, ${ }^{*} p<0.05$; compared with H group, ${ }^{*} p<0.05$; compared with HS group, ${ }^{\wedge} p<0.05$. 

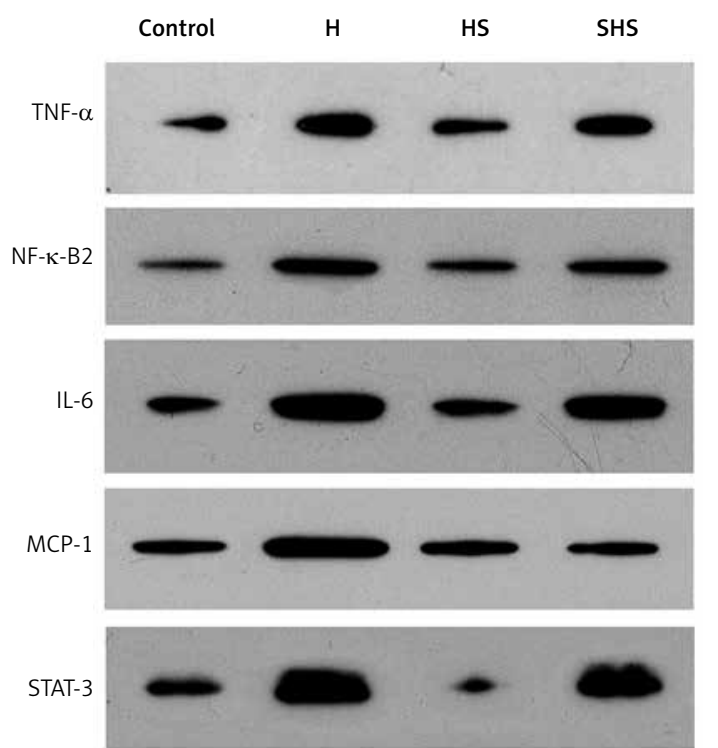

GADPH
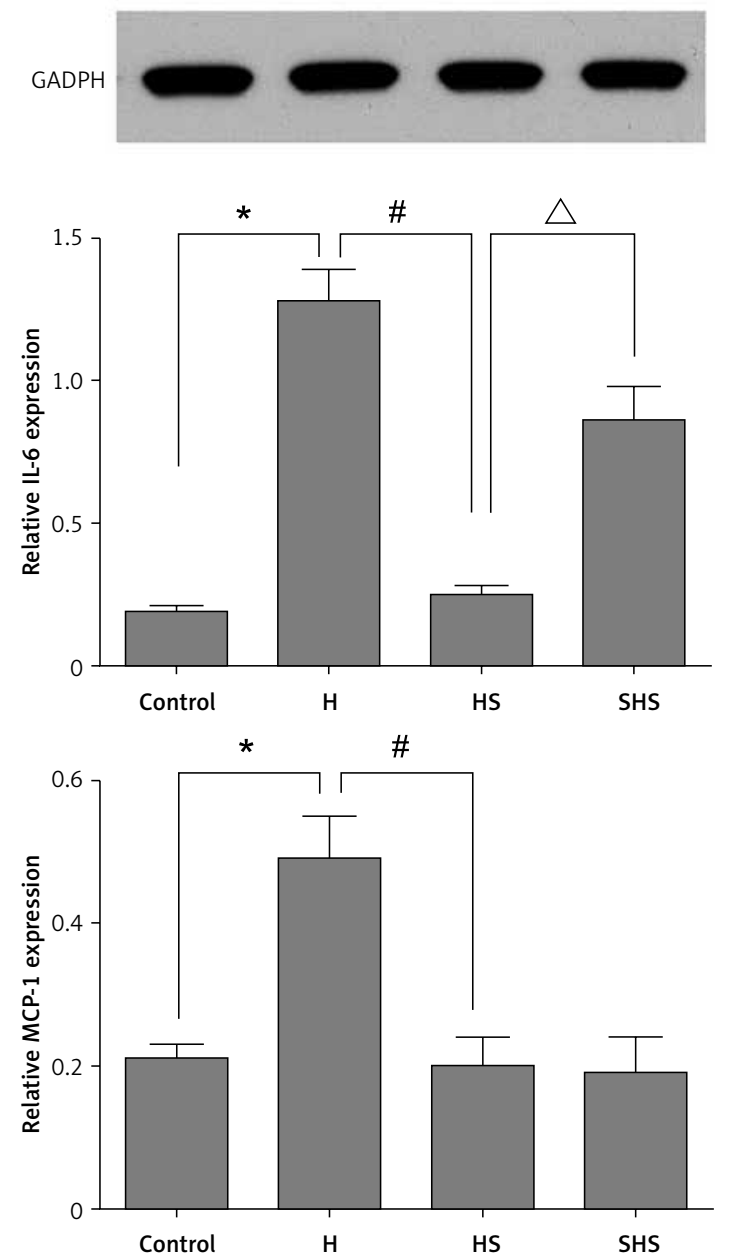
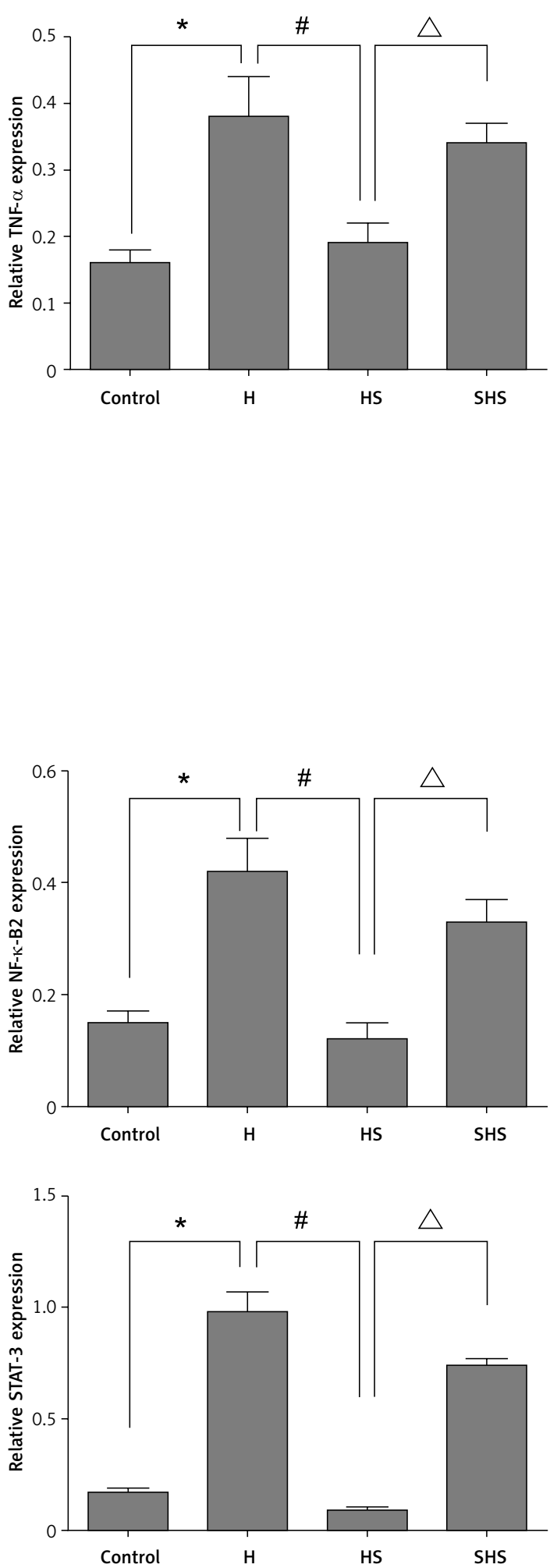

Figure 8. Expression of IL-6, TNF- $\alpha$, MCP-1, STAT-3 and NF- $\kappa-B 2$ with STAT-3 gene silencing in T cells

$H$ - hypoxic condition, HS - hypoxic condition plus Sal, SHS - hypoxic condition plus Sal after STAT-3 gene silencing. Compared with the control group, ${ }^{*} p<0.05$; compared with H group, ${ }^{*} p<0.05$; compared with HS group, ${ }^{\Delta} p<0.05$. 
In conclusion, salidroside can reduce the neurological score of MCAO model rats, reduce the volume of cerebral infarction and have neuroprotective effects. This effect may be related to inhibition of the expression of IL-6, TNF- $\alpha$, MCP-1, STAT-3 and NF-K-B2. STAT-3 plays an important role in this process.

\section{Acknowledgments}

This work was supported by the Fundamental Research Funds for the Central Universities (No. 21614311), the Natural Science Foundation of Guangdong Province, China (No. 2015A030310231), the National Natural Science Foundation of China (No. 81201016 and No. 81272027).

\section{Conflict of interest}

The authors declare no conflict of interest.

\section{References}

1. Reynolds MR, Derdeyn CP, Jr Grubb RL, et al. Extracranial-intracranial bypass for ischemic cerebrovascular disease: what have we learned from the Carotid Occlusion Surgery Study? Neurosurg Focus 2014; 36: E9.

2. Kong Q, Ma X. Contributing mechanisms of aortic atheroma in ischemic cerebrovascular disease. I Stroke Cerebrovasc Dis 2015; 24: 2653-9.

3. López M, Dávalos A, López M, et al. Advances in cerebrovascular disease research in the last year. J Neurol 2011; 258: 168-72.

4. Chen SD, Yin JH, Hwang CS, et al. Yang. Anti-apoptotic and anti-oxidative mechanisms of minocycline against sphingomyelinase/ceramide neurotoxicity: implication in Alzheimer's disease and cerebral ischemia. Free Radic Res 2012; 46: 940-50.

5. Panickar KS, Anderson RA. Effect of polyphenols on oxidative stress and mitochondrial dysfunction in neuronal death and brain edema in cerebral ischemia. Int J Mol Sci 2011; 12: 8181-207.

6. Antonysamy MA, Fanslow WC, Fu F, et al. Evidence for a role of IL-17 in organ allograft rejection: IL-17 promotes the functional differentiation of dendritic cell progenitors. J Immunol 1999; 162: 577-84.

7. Grund LZ, Novaski I, Quesniaux VF, et al. Neutrophils releasing IL-17A into NETs are essential to plasma cell differentiation in inflamed tissue dependent on IL-1R. Autoimmunity 2017; 50: 86-101.

8. Schön MP. The plot thickens while the scope broadens: a holistic view on IL-17 in psoriasis and other inflammatory disorders. Exp Dermatol 2014; 23: 804-6.

9. Korn T, Bettelli E, Oukka M, et al. IL-17 and Th17 cells. Annu Rev Immunol 2009; 27: 485-517.

10. Palomares O, Martín-Fontecha M, Lauener R, et al. Regulatory $T$ cells and immune regulation of allergic diseases: roles of IL-10 and TGF-beta. Genes Immun 2014; 15: 511-20.

11. Barbi J, Pardoll DM, Pan F. Ubiquitin-dependent regulation of Foxp3 and Treg function. Immunol Rev 2015; 26 627-45.

12. Morikawa H, Sakaguchi S. Genetic and epigenetic basis of Treg cell development and function: from a FoxP3-centered view to an epigenome-defined view of natural Treg cells. Immunol Rev 2014; 259: 192-205.

13. Nisar A, Akhter N, Singh G, et al. Modulation of T-helper cytokines and inflammatory mediators by Atropa accuminata. Royle in adjuvant induced arthritic tissues. J Ethnopharmacol 2015; 162: 215-24.

14. Kumar P, Natarajan K, Shanmugam N. High glucose driven expression of pro-inflammatory cytokine and chemokine genes in lymphocytes: molecular mechanisms of IL17 family gene expression. Cell Signal 2014; 26: 528-39.

15. Hot A, Lavocat $F$, Lenief $V$, et al. Simvastatin inhibits the pro-inflammatory and pro-thrombotic effects of IL17 and TNF-alpha on endothelial cells. Ann Rheum Dis 2013; 72: 754-60.

16. Lin CC, Chen DY, Tang KT, et al. Inhibitory effects of propofol on Th17 cell differentiation. Immunopharmacol Immunotoxicol 2017; 9: 211-8.

17. Titomanlio F, Perfumi M, Mattioli L, et al. Extract and its active compound salidroside antagonized both induction and reinstatement of nicotine place preference in mice. Psychopharmacology (Berl) 2014; 231: 2077-86.

18. Han J, Xiao Q, Lin YH, et al. Neuroprotective effects of salidroside on focal cerebral ischemia/reperfusion injury involve the nuclear erythroid 2-related factor 2 pathway. Neural Regen Res 2015; 10: 1989-96.

19. Longa EZ, Weinstein PR, Carlson S, et al. Reversible middle cerebral artery occlusion without craniectomy in rats. Stroke 1989; 20: 84-91.

20. Bleilevens C, Roehl AB, Goetzenich A, et al. Effect of anesthesia and cerebral blood flow on neuronal injury in a rat middle cerebral artery occlusion (MCAO) model. Exp Brain Res 2013; 224: 155-64.

21. Xiao S, Jin H, Korn T, et al. Retinoic acid increases Foxp3+ regulatory $T$ cells and inhibits development of Th17 cells by enhancing TGF-beta-driven Smad3 signalling and inhibiting IL- 6 and IL-23 receptor expression. J Immunol 2008; 181: 2277-84.

22. Park JS, Kwok SK, Lim MA, et al. STA-21, a promising STAT-3 inhibitor that reciprocally regulates Th17 and Treg cells, inhibits osteoclastogenesis in mice and humans and alleviates autoimmune inflammation in an experimental model of rheumatoid arthritis. Arthritis Rheumatol 2014; 66: 918-29.

23. Tao XY, Wang L, Gao WY, et al. The effect of inducible nitric oxide synthase on multiterritory perforator flap survival in rats. J Reconstr Microsurg 2016; 32: 643-9.

24. Liu S, Yu X, Hu B, et al. Salidroside rescued mice from experimental sepsis through anti-inflammatory and anti-apoptosis effects. J Surg Res 2015; 195: 277-83.

25. Fang DL, Chen Y, Xu B, et al. Development of lipid-shell and polymer core nanoparticles with water-soluble salidroside for anti-cancer therapy. Int J Mol Sci 2014; 15: 3373-88.

26. Zheng Y, Sun L, Jiang T, et al. TNFalpha promotes Th17 cell differentiation through IL- 6 and IL-1beta produced by monocytes in rheumatoid arthritis. J Immunol Res 2014; 2014: 385352.

27. McHugh MD, Park J, Uhrich R, et al. Paracrine co-delivery of TGF-beta and IL-2 using CD4-targeted nanoparticles for induction and maintenance of regulatory $T$ cells. Biomaterials 2015; 59: 172-81.

28. Jhunjhunwala S, Balmert SC, Raimondi G, et al. Controlled release formulations of IL-2, TGF-beta1 and rapamycin for the induction of regulatory $T$ cells. J Control Release 2012; 159: 78-84.

29. Murawski MR, Litherland SA, Clare-Salzler MJ, DavoodiSemiromi A. Upregulation of Foxp3 expression in mouse 
and human Treg is IL-2/STAT5 dependent: implications for the NOD STAT5B mutation in diabetes pathogenesis. Ann N Y Acad Sci 2006; 1079: 198-204.

30. Burchill MA, Yang J, Vogtenhuber C, et al. IL-2 receptor beta-dependent STAT5 activation is required for the development of Foxp3+ regulatory T cells. J Immunol 2007; 178: 280-90.

31. Goodman WA, Cooper KD, McCormick TS. Regulation generation: the suppressive functions of human regulatory T cells. Crit Rev Immunol 2012; 32: 65-79.

32. Wang GQ, Yang CL, Yue DF, et al. The changes and its significance of Th17 and Treg cells and related cytokines in patients with tuberculosis pleurisy. Allergy Asthma Clin Immunol 2014; 10: 28.

33. Zheng Y, Wang Z, Deng L, et al. Modulation of STAT3 and STAT5 activity rectifies the imbalance of Th17 and Treg cells in patients with acute coronary syndrome. Clin Immunol 2015; 157: 65-77.

34. Yang XP, Ghoreschi K, Steward-Tharp SM, et al. Opposing regulation of the locus encoding IL-17 through direct, reciprocal actions of STAT3 and STAT5. Nat Immunol 2011; 12: 247-54. 